\title{
Terahertz Bandpass Frequency Selective Surfaces on Glass Substrates Using a Wet Micromachining Process
}

\author{
Mehrab Ramzan ${ }^{1}$ • Talha Masood Khan ${ }^{2} \cdot \operatorname{Sami}_{\text {Bolat }}{ }^{1}$ • \\ Mehmet Ali Nebioglu ${ }^{3} \cdot$ Hakan Altan ${ }^{3}$. \\ Ali Kemal Okyay ${ }^{1,2} \cdot$ Kagan Topalli ${ }^{2,4}$ if
}

Received: 13 February 2017 / Accepted: 11 May 2017 /

Published online: 1 June 2017

(C) Springer Science+Business Media New York 2017

\begin{abstract}
This paper presents terahertz (THz) frequency selective surfaces (FSS) implemented on glass substrate using standard microfabrication techniques. These FSS structures are designed for frequencies around $0.8 \mathrm{THz}$. A fabrication process is proposed where a 100 - $\mu \mathrm{m}$-thick glass substrate is formed through the HF etching of a standard $500-\mu \mathrm{m}$-thick low cost glass wafer. Using this fabrication process, three separate robust designs consisting of single-layer FSS are investigated using high-frequency structural simulator (HFSS). Based on
\end{abstract}

Kagan Topalli

kagan.topalli@gmail.com

Mehrab Ramzan

mehrabramzan@bilkent.edu.tr

Talha Masood Khan

talha.khan@bilkent.edu.tr

Sami Bolat

bolat@ee.bilkent.edu.tr

Mehmet Ali Nebioglu

mehmetalinebioglu@gmail.com

Hakan Altan

haltan@metu.edu.tr

Ali Kemal Okyay

aokyay@gmail.com

1 Department of Electrical and Electronics Engineering, Bilkent University, 06800 Ankara, Turkey

2 UNAM-Institute of Materials Science and Nanotechnology, Bilkent University, 06800 Ankara, Turkey

3 Department of Physics, Middle East Technical University, 06800 Ankara, Turkey

4 TUBITAK Space Technologies Research Institute, 06800 Ankara, Turkey 
the simulation results, the first design consists of a circular ring slot in a square metallic structure on top of a 100- $\mu$ m-thick Pyrex glass substrate with $70 \%$ transmission bandwidth of approximately $0.07 \mathrm{THz}$, which remains nearly constant till $30^{\circ}$ angle of incidence. The second design consists of a tripole structure on top of a 100- $\mu$ m-thick Pyrex glass substrate with $65 \%$ transmission bandwidth of $0.035 \mathrm{THz}$, which remains nearly constant till $30^{\circ}$ angle of incidence. The third structure consists of a triangular ring slot in a square metal on top of a $100-\mu \mathrm{m}$-thick Pyrex glass substrate with $70 \%$ transmission bandwidth of $0.051 \mathrm{THz}$, which remains nearly constant up to $20^{\circ}$ angle of incidence. These designs show that the reflections from samples can be reduced compared to the conventional sample holders used in $\mathrm{THz}$ spectroscopy applications, by using single layer FSS structures manufactured through a relatively simple fabrication process. Practically, these structures are achieved on a fabricated $285-\mu \mathrm{m}$-thick glass substrate. Taking into account the losses and discrepancies in the substrate thickness, the measured results are in good agreement with the electromagnetic simulations.

Keywords Frequency selective surfaces · Bandpass filters · Periodic structures · Metamaterials · Microfabrication $\cdot$ HF etching $\cdot$ Pyrex glass $\cdot$ Terahertz

\section{Introduction}

In recent years, $\mathrm{THz}$ region has gained great attention due to its useful applications in the medical, space sciences, security, communication, and sensing fields [1-8]. There are numerous $\mathrm{THz}$ imaging devices commercially available, but the deficiency of robust, compact and smartly developed imaging tools is still a major concern. The low power output of sources available in the $\mathrm{THz}$ spectrum brings some challenges in $\mathrm{THz}$ imaging and spectroscopy [9-12]. In these measurements, reflections from the samples such as biological tissues, liquids, and powdered specimens placed in a sample holder are needed to be minimized in order to enhance the signal received from the sample itself. The commonly available glass slide sample holders have severe power losses. Figure 1 shows the reflection measurement setup from a biological tissue sample. In the case of using a glass slider as a specimen holder, much of the incident power is lost before the sample due to unavoidable reflections at the air-glass interface (Fig. 1a). Moreover, the thickness of FSS is also critical in order to minimize the substrate losses. Figure 2 shows the transmission coefficient comparison of bare $500-\mu \mathrm{m}$-thick Pyrex

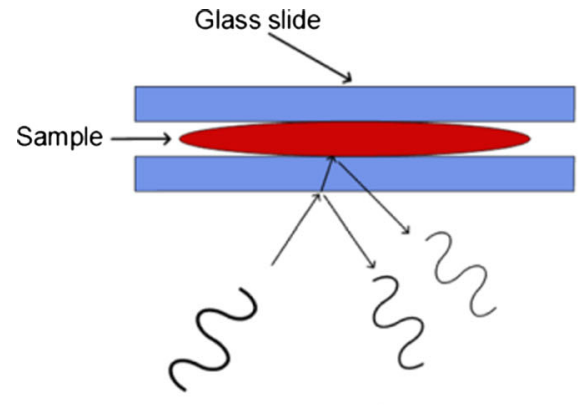

(a)

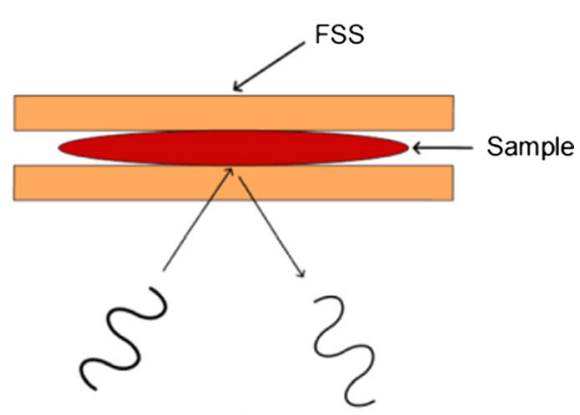

(b)

Fig. 1 Typical measurement setup of the reflections from a biological tissue. a Glass slide as a sample holder. b FSS as a sample holder 
Fig. 2 Transmission coefficient comparison of standard bare 500 $\mu \mathrm{m}$-thick Pyrex glass and 100- $\mu \mathrm{m}$ thick Pyrex glass with FSS

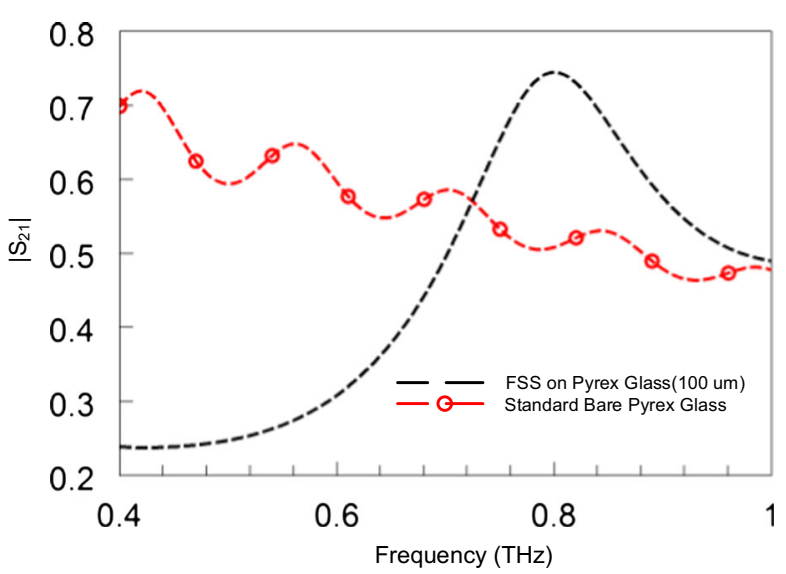

glass and 100- $\mu \mathrm{m}$-thick Pyrex glass with FSS. Therefore, proper thinning of glass holders with mechanical support and selection of FSS structures with transparent qualities can play a very vital role to match the impedance of the device to air in order to suppress the unavoidable
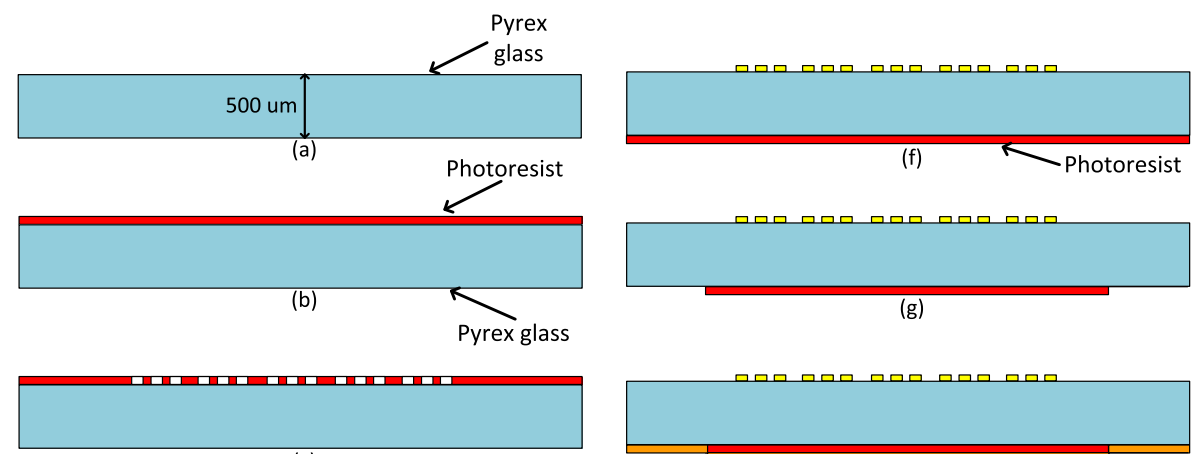

(c)

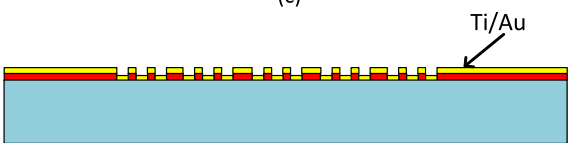

(d)

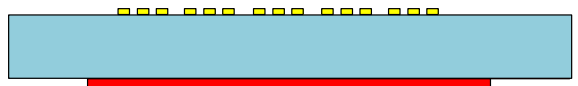

(g)

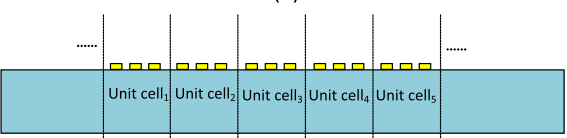

(e)



(i)

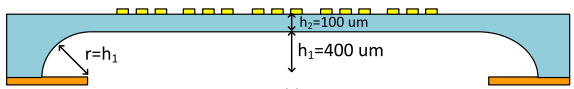

(j)

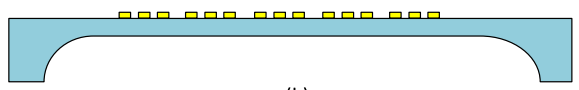

(k)

Fig. 3 Fabrication process flow. a A 500- $\mu$ m-thick Pyrex glass. b Spin coating of photoresist. c Patterning the photoresist for liftoff process. d Deposition of the metal. e After liftoff only unit cell patterns are left. f Spin coating of the photoresist. g Patterning the photoresist for the liftoff process. h Deposition of $\mathrm{Cr} / \mathrm{Au} / \mathrm{Cr} / \mathrm{Au}$. i Liftoff process using acetone. $\mathbf{j}$ Wet etching of the lower side of Pyrex glass using HF while the other side is protected with a wafer holder. $\mathbf{k} 100-\mu \mathrm{m}$-thick Pyrex glass substrate with metal patterns on top of it 
Fig. 4 HFSS unit cell with master slave boundary conditions and Floquet ports for simulating periodic structures

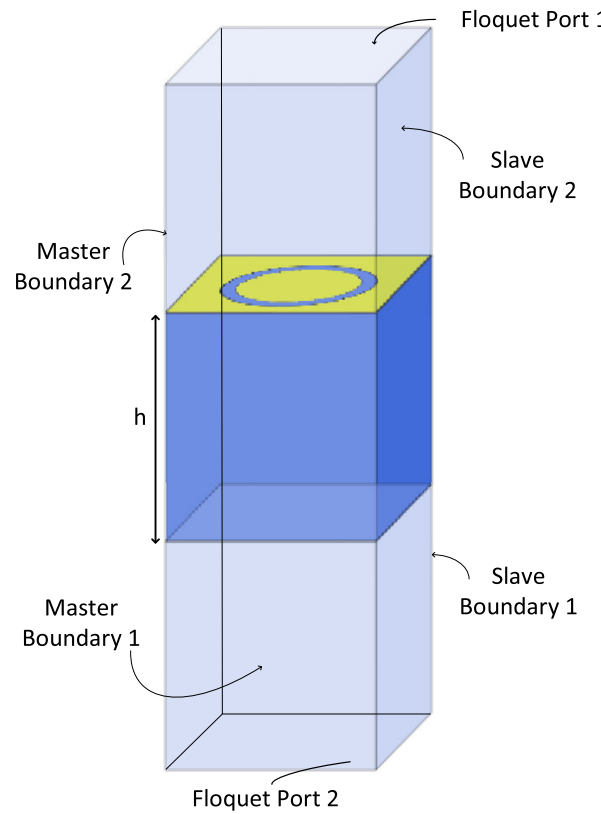

reflections without compromising much of the incident power of the radiation (Fig. 1b). The design of these patterns requires special care, such that they should have minimum reflections, maximum transmission, and at the same time their response should be independent of certain angles of incidence [13-16]. There are most studies about multilayer bandpass frequency

(i)
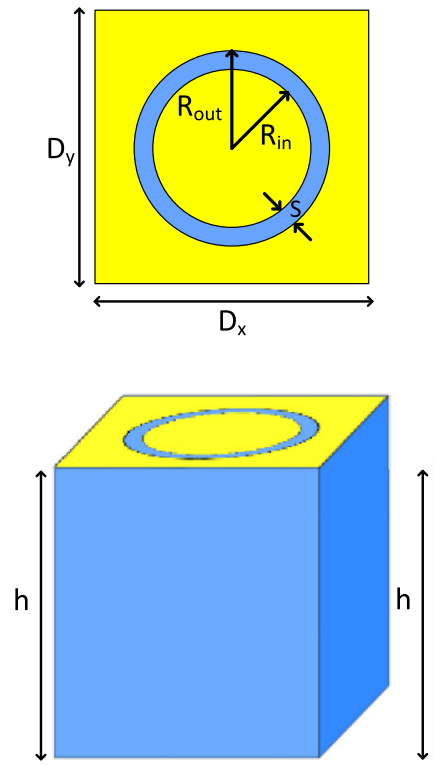

(ii)
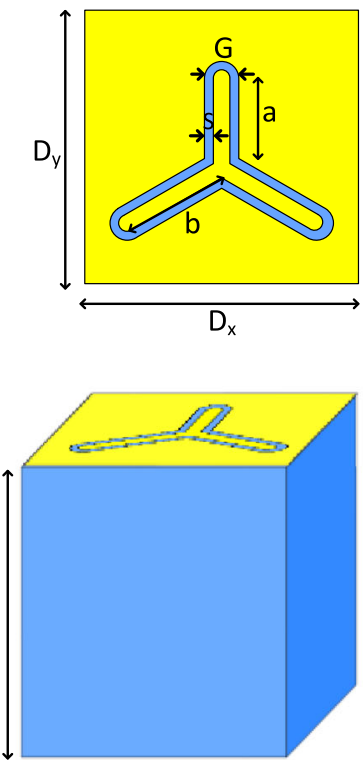

(iii)
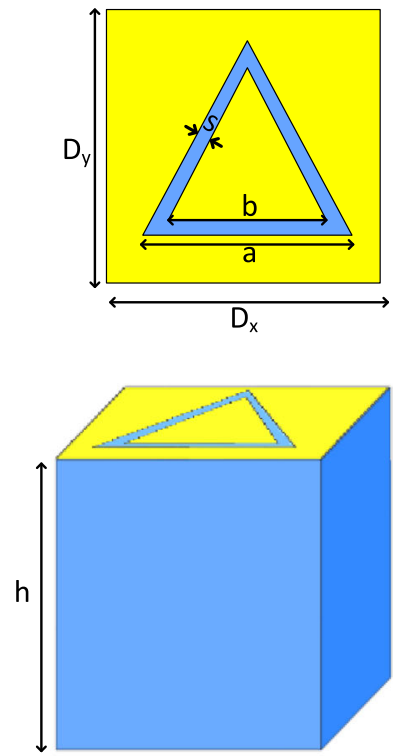

Fig. 5 Unit cell parameters of the (i) circular slot FSS, (ii) tripole slot FSS, and (iii) triangular slot FSS 
Table 1 Parameters of the circular, tripole, and triangular slot FSS structures

\begin{tabular}{cclclc}
\hline Circular $(\mu \mathrm{m})$ & \multicolumn{2}{c}{ Tripole $(\mu \mathrm{m})$} & \multicolumn{2}{c}{ Triangle $(\mu \mathrm{m})$} \\
\hline $\mathrm{D}_{\mathrm{x}}$ & 100 & $\mathrm{D}_{\mathrm{x}}$ & 100 & $\mathrm{D}_{\mathrm{x}}$ & 100 \\
$\mathrm{D}_{\mathrm{y}}$ & 100 & $\mathrm{D}_{\mathrm{y}}$ & 100 & $\mathrm{D}_{\mathrm{y}}$ & 100 \\
$\mathrm{R}_{\text {in }}$ & 37 & $\mathrm{a}$ & 37.8 & $\mathrm{a}$ & $\mathrm{b}$ \\
$\mathrm{R}_{\text {out }}$ & 40 & $\mathrm{~b}$ & 37.8 & $\mathrm{~S}$ & 81.53 \\
$\mathrm{~S}$ & 3 & $\mathrm{~S}$ & 3.5 & $\mathrm{~h}$ & 3 \\
$\mathrm{~h}$ & 100 & $\mathrm{~h}$ & 100 & - & 100 \\
- & - & $\mathrm{G}$ & 10 & & - \\
\hline
\end{tabular}

selective surfaces which have high complexity and require high precision of microfabrication such as multilayer bonding, alignment of top and bottom layer elements, etc. [17-19]. This study demonstrates the use of simple single layer FSS designs in the THz region, which have more than $70 \%$ transmission at the central frequency independent of various angles of incidence. In order to model the simulation results perfectly in the $\mathrm{THz}$ region, the precise calculation of the loss tangent, $\tan \delta$, plays a very important role before fabrication. Additionally, an acceptable design membrane fabrication process is proposed which makes this idea more applicable. In this paper, using the given value of $\tan \delta$, the simulated model of the FSS using HFSS results is in perfect agreement with the measurement results.

\section{Microfabrication of FSS}

A cost effective and robust microfabrication process is proposed for designing FSS in $\mathrm{THz}$ regime. A standard low cost Pyrex glass is used as the substrate with a permittivity of 4.45 and $\tan \delta$ of 0.058 which is extracted from [9]. Pyrex glass is commercially available with a

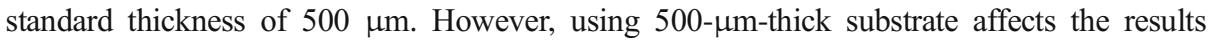
significantly by causing the excitation of substrates modes. To this end, substrate is etched from beneath to the required design thickness of $100 \mu \mathrm{m}$. In order to provide mechanical support to the substrate, the periphery of FSS is kept at $500 \mu \mathrm{m}$ thickness. To realize the designs, a fabrication process flow is designed as given below.

Fig. 6 Array of circular ring slot in a square metal on top of 100$\mu \mathrm{m}$-thick Pyrex glass substrate




Fig. 7 Array of tripole slot in a square metal on top of a $100-\mu \mathrm{m}-$ thick Pyrex glass substrate



Initially on a 500- $\mu$ m-thick Pyrex glass (Fig. 3a), the patterns are generated in the area in between the supporting anchor points of the substrate. Photoresist is coated on top of the substrate (Fig. 3b). Liftoff process is used for pattern generation, the photoresist is exposed to UV light with the mask aligner and left in developer to make empty spaces for pattern generation (Fig. 3c). After the lithography process, $10 \mathrm{~nm}$ of $\mathrm{Ti}$ is used as an adhesion layer, followed by $250 \mathrm{~nm}$ of $\mathrm{Au}$ deposited on top of the substrate using e-beam evaporation (Fig. 3d). In order to remove the unwanted metal on top of photoresist, the sample is kept in acetone which lifts off all the unwanted metal. Finally, the sample top is left with the array patterns of the unit cells (Fig. 3e).

After pattern generation, the thickness of the glass is reduced to $100 \mu \mathrm{m}$ using wet etching with hydrofluoric acid (HF). Since HF is very active against photoresist, it cannot be used as a

Fig. 8 Array of triangular slot in a square metal on top of a $100-\mu \mathrm{m}-$ thick Pyrex substrate

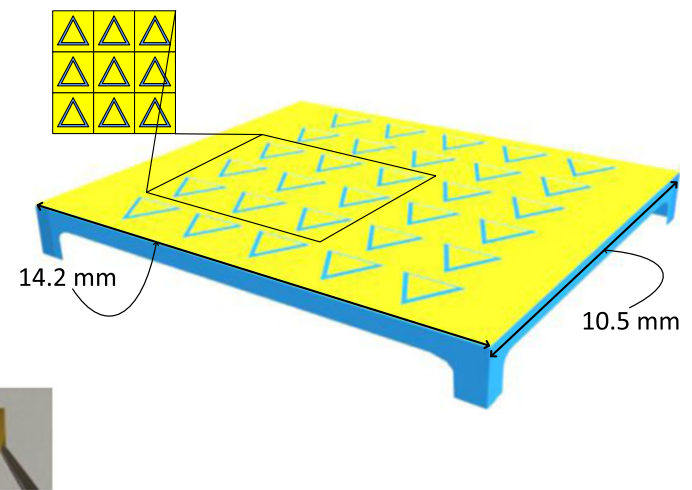


Fig. 9 HFSS simulation results of the transmission coefficient of the single layer bandpass circular slot FSS filter inside a square metal for TE case at different angle of incidence

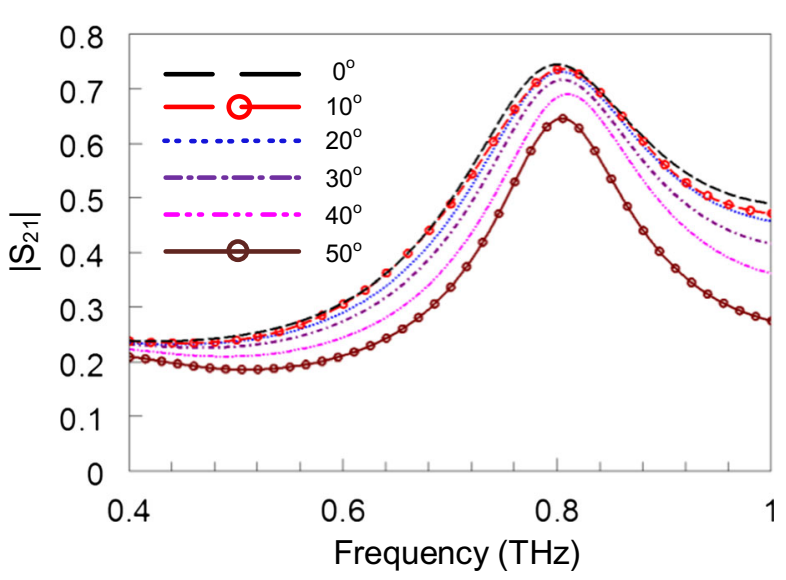

protection layer against the glass etching process. For Pyrex glass etching, $\mathrm{Cr} / \mathrm{Au} / \mathrm{Cr} / \mathrm{Au}$ with the corresponding thickness of 65/350/65/350 nm layers are used as a masking layer to protect the anchors from etching. Double deposition of $\mathrm{Cr} / \mathrm{Au}$ layer improves the strength of the masking for against HF wet etching of Pyrex glass. Patterns are defined with the photoresist using double sided alignment process (Fig. 3f). After that, using a mask aligner and developing methods, photoresist is removed from the regions to be protected in glass etching (Fig. 3g). Cr/ $\mathrm{Au} / \mathrm{Cr} / \mathrm{Au}$ are deposited (Fig. 3h). The sample is kept in acetone for lifting-off the unwanted metal deposited on the top of photoresist (Fig. 3i). After the lift-off process, only the glass anchor region to be protected are covered with $\mathrm{Cr} / \mathrm{Au} / \mathrm{Cr} / \mathrm{Au}$ mask. The other surface of the wafer is protected from HF etching using a wafer holder. As HF-dip is a wet etching process, the etching profile will be isotropic. After $1 \mathrm{~h}$ of the glass etching in HF, a $100-\mu \mathrm{m}$ thick glass substrate is left beneath the unit cells (Fig. 3j). Finally, $\mathrm{Cr} / \mathrm{Au} / \mathrm{Cr} / \mathrm{Cr}$ layers are removed using $\mathrm{Cr} / \mathrm{Au}$ etchants and 500- $\mu \mathrm{m}$-thick anchor points are left at the edges of the substrates which will give mechanical support to the end product and make the design more robust (Fig. 3k).

Fig. 10 HFSS simulation results of the transmission coefficient of the single layer bandpass circular slot FSS filter inside a square metal for TM case at different angle of incidence

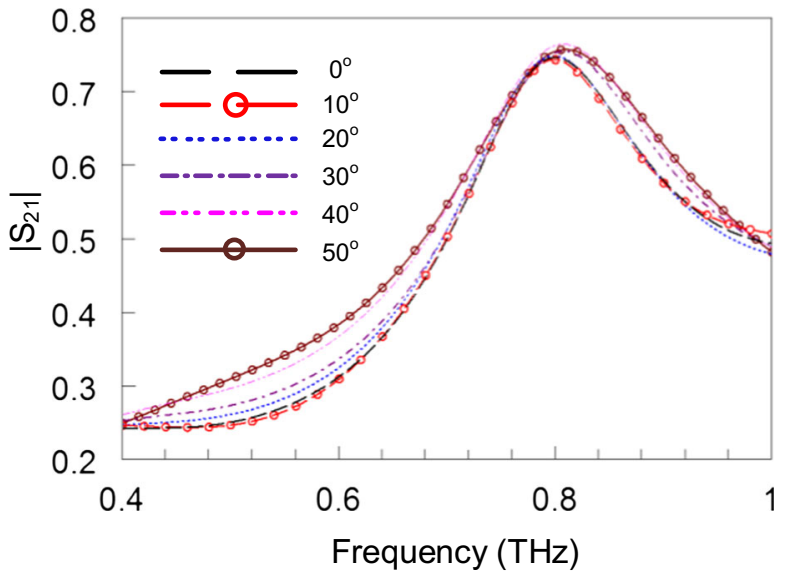


Fig. 11 HFSS simulation results of the transmission coefficient of the single layer tripole slot FSS filter inside a square metal for TE case at different angle of incidence

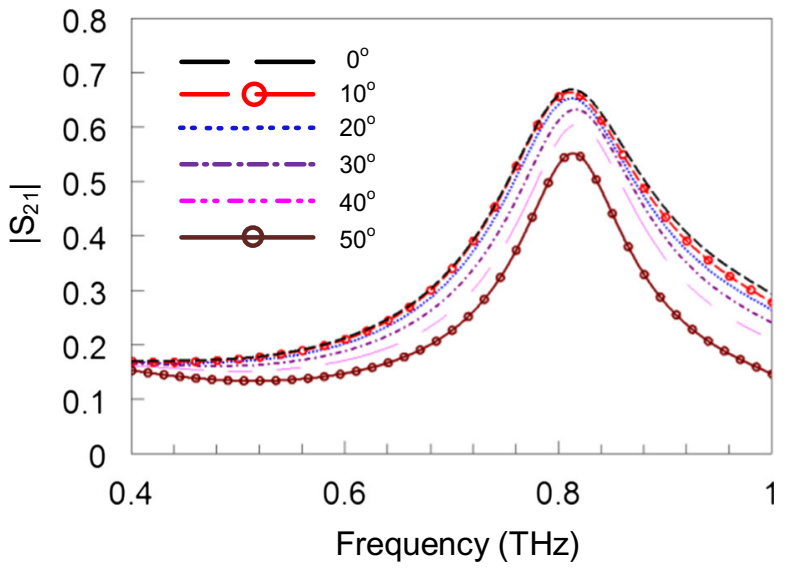

\section{A 3-Dimensional Full Wave EM Modeling Approach}

High frequency structural simulator (HFSS) is used for the FSS device simulations. In HFSS, periodic boundary conditions such as master and slave are used for simulating the periodic structures with the Floquet port excitation as shown in Fig. 4. Master and slave boundary conditions model the plane of periodicity, where E-field on one surface matches the E-field to another within a phase difference [20]. Based on the defined microfabrication process, three different FSS structures with bandpass characteristics are designed. The first structure consists

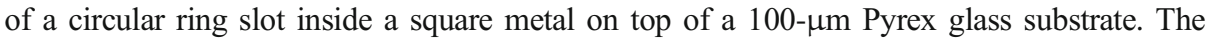
second structure is the tripole slot inside a square metal with bandpass characteristics. The third structure consists of a triangular slot inside a square metal. The unit cells on Pyrex glass are shown in Fig. 5. Table 1 shows the unit cell dimensions of circular, tripole and triangular slot, respectively.

Figures 6,7 , and 8 show the FSS structures consisting of circular, tripole and triangular slots, respectively. The FSS structures have $100 \times 100$ unit cells with four supporting anchor

Fig. 12 HFSS simulation results of the transmission coefficient of the single layer tripole slot FSS filter inside a square metal for TM case at different angle of incidence

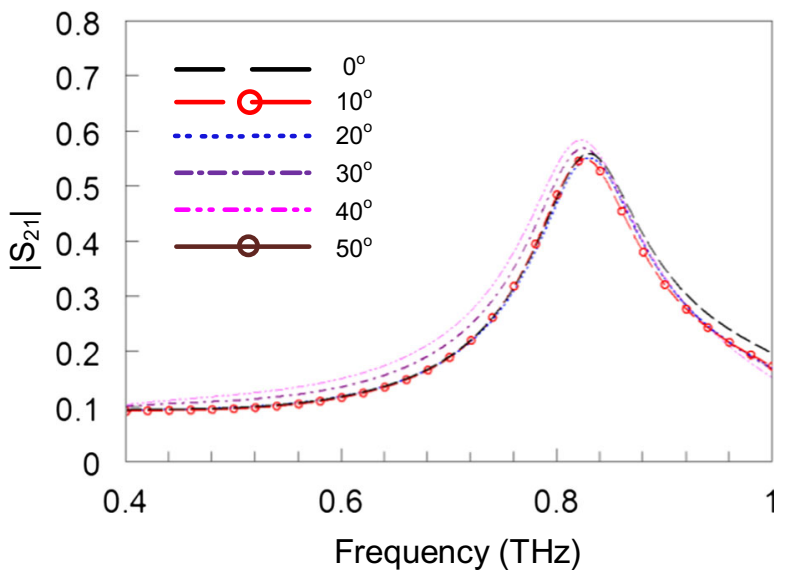


Fig. 13 HFSS simulation results of the transmission coefficient of the single layer bandpass triangular slot FSS filter inside a square metal for TE case at different angle of incidence

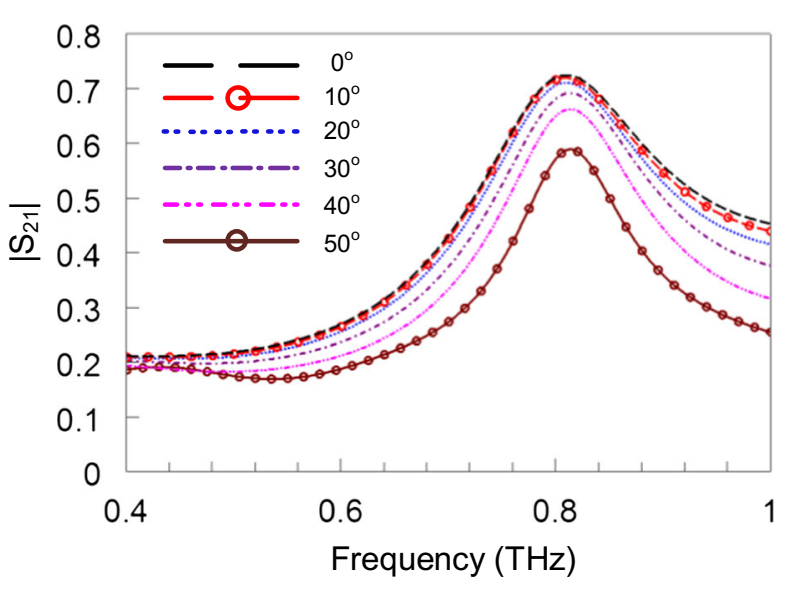

points at the edges. Figures 9 and 10 show the circular ring slot array simulation results for TE and TM wave configurations, respectively. The center frequency is $0.8 \mathrm{THz}$ with maximum transmission coefficient of $74 \%$. The $70 \%$ transmission bandwidth of this device is $0.07 \mathrm{THz}$ and its results are nearly independent till $30^{\circ}$ of angle of incidence. Figures 11 and 12 show simulation results of tripole slots with center frequency around $0.8 \mathrm{THz}$ for TE and TM cases, respectively. The maximum transmission for TE and TM case are 67 and $60.6 \%$, respectively. The results for this structure remain nearly same up to $30^{\circ}$ of angle of incidence. However, the losses in this structure are higher and it shows the top structure selection also plays a vital role in deciding the maximum transmission through the device. Figures 13 and 14 show transmission coefficient simulation results of triangular slots for TE and TM cases, respectively, with maximum transmission of $73 \%$ at $0.8 \mathrm{THz}$. The $70 \%$ bandwidth of triangle slot is $0.05 \mathrm{THz}$ and remains nearly constant up to $20^{\circ}$ angle of incidence. It should be noted here that independent of the unit cell geometry, either it is circular, triangular or tripole, the FSS itself provides an enhancement yielding a bandpass characteristics within a certain band.

Fig. 14 HFSS simulation results of the transmission coefficient of the single layer bandpass triangular slot FSS filter inside a square metal for TM case at different angle of incidence

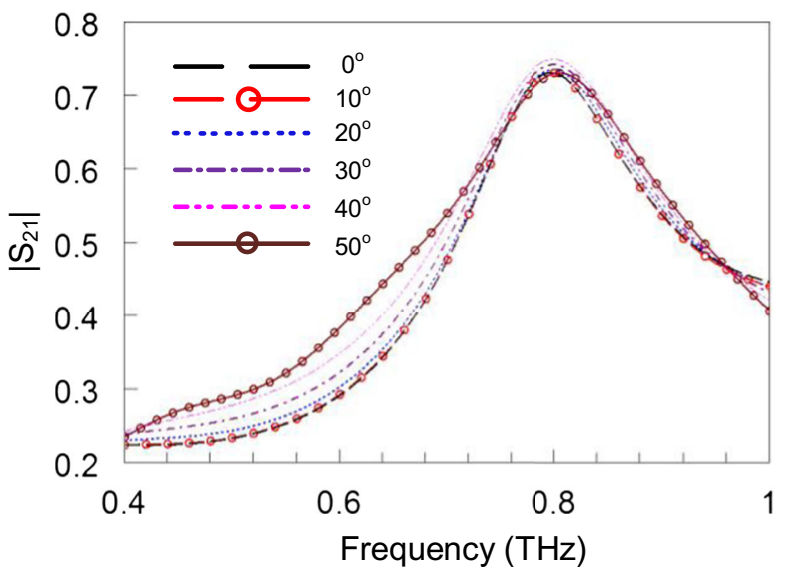


Fig. 15 Comparison of measured and simulated results of the circular ring slots on top of a Pyrex glass substrate for TE polarized wave

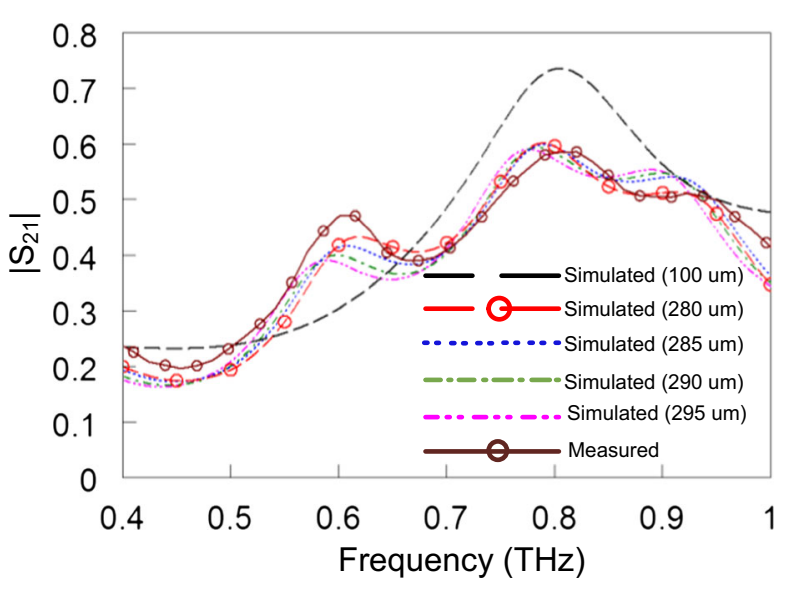

\section{Measurement Results and Discussion}

In order to measure the transmission properties of the FSS structures, a home built THz-TDS system is used. A Ti: $\mathrm{Al}_{2} \mathrm{O}_{3}$ mode-lock laser operating at a center wavelength of $800 \mathrm{~nm}$, a pulse duration of $16 \mathrm{f}$. and repetition rate of $75 \mathrm{MHz}$ with an average power $\sim 200 \mathrm{~mW}$ are employed to drive the system. A beam splitter with a 95/5 of transmission ratio splits the incoming beam into the two optical arms of the spectrometer. The polarization of the generated $\mathrm{THz}$ beam is perpendicular to the horizontal plane (s-polarization). The $\mathrm{THz}$ radiation is generated using a photoconductive antenna and is guided through the system using two offaxis parabolic mirrors and two lenses. The two lenses are similar (TPX lens, $D=50 \mathrm{~mm}, \mathrm{F \# 2}$ ) and are used to focus the $\mathrm{THz}$ beam onto the sample with a spot diameter of about $5 \mathrm{~mm}$. Afterwards, the electric field of the $\mathrm{THz}$ pulse is measured using electro-optic detection method through a balanced photodiode. Phase sensitive detection is done by use of a lock-in amplifier which is referenced to an applied AC bias on the photoconductive antenna. The data is recorded with the aid of a PC and data acquisition software.

Fig. 16 Comparison of measured and simulated results of the tripole slots on top of a Pyrex glass substrate for TE polarized wave

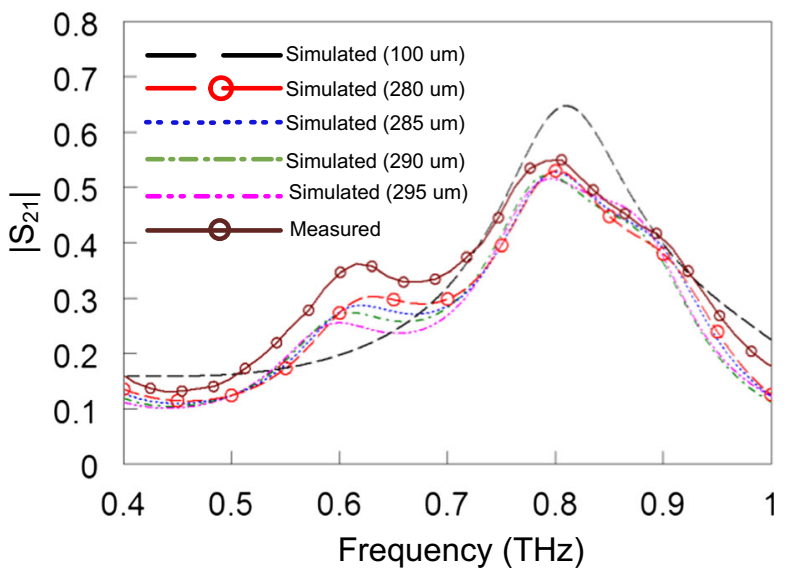


Fig. 17 Comparison of measured and simulated results of the triangular slots on top of a Pyrex glass substrate for TE polarized wave

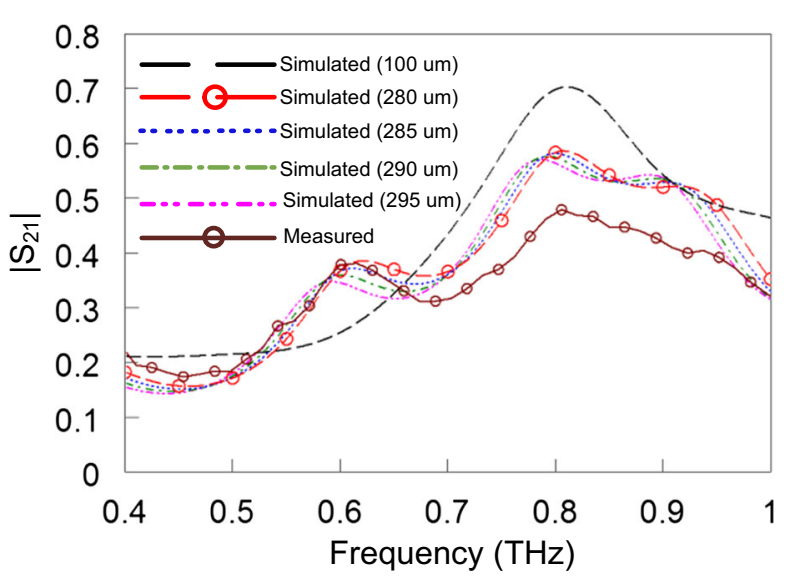

In $\mathrm{THz}$ region in order to have a precise match between the simulation and measurement results special care has to be taken for the $\tan \delta$ calculations and final substrate thickness. Figures 15, 16, and 17 show the comparison of measured and simulated transmission coefficient of circular, tripole and triangular ring slots, respectively, on top of the Pyrex glass substrate. The measured results in each case show a drop in the maximum peak and distortion in the shape of the transmission coefficient due to the insufficient etching of the Pyrex glass as shown in Fig. 18, the SEM image of cross-sectional view of the fabricated FSS substrate with the thickness of approximately $283 \mu \mathrm{m}$. Additional simulation results of glass substrates with thickness ranging from 280 to $295 \mu \mathrm{m}$ show that on average $285 \mu \mathrm{m}$ thickness value has a very good agreement with the measurement results. There are some discrepancies between the simulated and measured results of triangular slot case due to some fabrication tolerances and

Fig. 18 SEM image showing the cross-sectional view of the fabricated FSS substrate indicating a thickness of about $283 \mu \mathrm{m}$

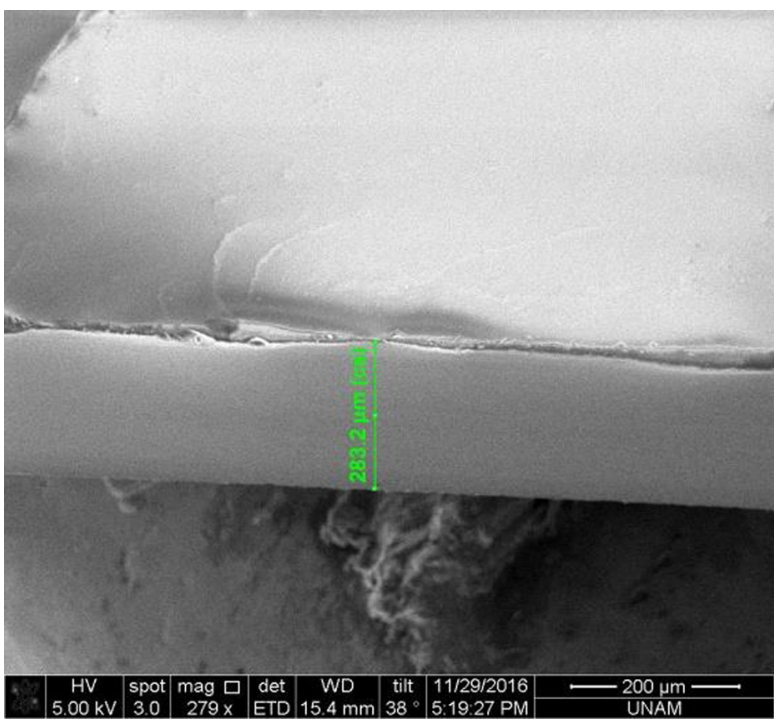


(a)

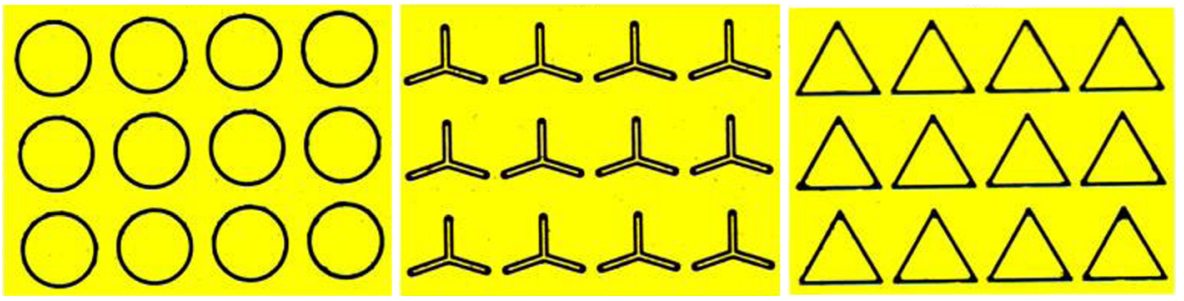

Fig. 19 Optical pictures of the fabricated array of $\mathbf{a}$ circular, $\mathbf{b}$ tripole, and $\mathbf{c}$ triangular slots on top of the Pyrex glass substrate

distortion of some of the $100 \times 100$ element array during the lift-off process. Figure 19a-c shows some optical images of the fabricated arrays of the circular ring, tripole and triangular slots, respectively.

\section{Conclusion}

We demonstrated a low cost and robust design methodology for implementing single layer, bandpass characteristic FSS structure with an acceptable level of loss. A full-fledged device consisting of $100 \times 100$ elements on a $285-\mu \mathrm{m}$-thick substrate with $500-\mu \mathrm{m}$-thick anchors at the periphery, are fabricated using a relatively simple microfabrication technique. The maximum transmission can be achieved by further thinning the substrate thickness to $100 \mu \mathrm{m}$. The simulated designs with $100 \mu \mathrm{m}$ demonstrate a transmission of about $75 \%$ at a center frequency of $0.8 \mathrm{THz}$. The losses can be reduced further by using a low-loss material such as fused silica at the expense of high cost and more complex fabrication processes that requires glass ablation process using femtosecond lasers.

Acknowledgments This work was partially supported by the Scientific and Technological Research Council of Turkey (TUBITAK) under Grant 113E126. Hakan Altan also acknowledges support from BAGEP Award of the Science Academy in Turkey.

\section{References}

1. P. H. Siegel, "Terahertz technology," IEEE Transactions on Microwave Theory and Techniques, 50, 3, pp. 910-928, 2002.

2. Y. Sun, M. Y. Sy, Y. -X. J. Wang, A. T. Ahuja and Y. T. Zhang, "A promising diagnostic method: Terahertz pulsed imaging and spectroscopy," World Journal of W J R Radiology, pp. 55-65, 2011

3. M. C. Kemp, P. F. Taday, B. E. Cole, J. A. Clu, A. J. Fitzgerald and W. R. Tribe, "Security applications of terahertz technology," Proc. of SPIE, 50, 3, pp. 44-52, 2003.

4. T. W. Crowe, T. Globus, D. L. Woolard and J. L. Hesler, "Terahertz sources and detectors and their application to biological sensing," Philosophical Transactions: Mathematical, Physical and Engineering Sciences, 362, 1815, pp. 365-377, 2004.

5. A. Redo-Sanchez and X. -C. Zhang, "Terahertz science and technology trends," IEEE Journal of Selected Topics in Quantum Electronics, 14, 2, pp. 260-269, March 2008.

6. C. Corsi and F. Sizov, THz and Security Applications: Detectors, Sources and Associated Electronics, Springer, 2014.

7. H.-B. Liu, H. Zhong, N. Karpowicz, Y. Chen and X.-C. Zhang, "Terahertz spectroscopy and Imaging for defense and security applications," Proceedings of the IEEE, 95, 8, pp. 1514-1527, August 2007. 
8. P. H. Siegel, "Terahertz technology in biology and medicine," IEEE Transactions on Microwave Theory And Techniques, 52, 10, pp. 2438-2447, 2004.

9. M. Naftaly and R. E. Miles, "Terahertz Time-Domain Spectroscopy for Material Characterization," Proceedings of the IEEE, 95, 8, August 2007.

10. V. Sanphuang, W. G. Yeo, J. L. Volakis and N. K. Nahar, "THz transparent metamaterials for enhanced spectroscopic and imaging measurements," IEEE Transactions on Terahertz Science And Technology, 5, pp. 117-122, January 2015.

11. E. Bründermann, H. -W. Hübers and M. F. Kimmitt, Terahertz Techniques, Springer, 2012.

12. G. Chattopadhyay, "Technology, Capabilities, and Performance of Low Power Terahertz Sources," IEEE Transactions on Terahertz. Science and Technology, 1, 1, September 2011.

13. S. Vegesna, Y. Zhu, A. Bernussi and M. Saed, "Terahertz two-layer frequency selective surfaces with improved transmission characteristics," IEEE Transactions on Terahertz Science And Technology, 2, pp. 441-448, July 2012.

14. B. A. Munk, Frequency selective surfaces theory and design, John Wiley \& Sons, 2000.

15. T. Wu, Frequency selective surface and grid array, John Wiley \& Sons, 1995.

16. R. Dickie, R. Cahill, V. Fusco, H. S. Gamble and N. Mitchell, "THz Frequency Selective Surface Filters for Earth Observation Remote Sensing Instruments," IEEE Transactions On Terahertz Science and Technology, 1, 2, November 2011.

17. D. S. Wang, B. J. Chen and C. H. Chan, "High-Selectivity Bandpass Frequency-Selective Surface in Terahertz Band," IEEE Transactions on Terahertz Science and Technology, 6, 2, March 2016.

18. A. Ebrahimi, S. Nirantar, W. Withayachumnankul, M. Bhaskaran, S. Sriram, S. F. Al-Sarawi and D. Abbott, "Second-Order Terahertz Bandpass Frequency Selective Surface With Miniaturized Elements," IEEE Transaction on Terahertz Science And Technology, 5, 5, September 2015.

19. S. M. A. M. H. Abadi, M. Li, and N. Behdad, "Harmonic-Suppressed Miniaturized-Element Frequency Selective Surfaces With Higher Order Bandpass Responses," IEEE Transactions on Antennas and Propagation, 62, 5, May 2014.

20. Ansoft, user's guide - High Frequency Structure Simulator, Ansoft Corporation, 2005. 